\title{
YELLOW FEVER VACCINE-ASSOCIATED NEUROLOGIC DISEASE IN SÃO PAULO, BRAZIL: REPORT OF TWO CASES
}

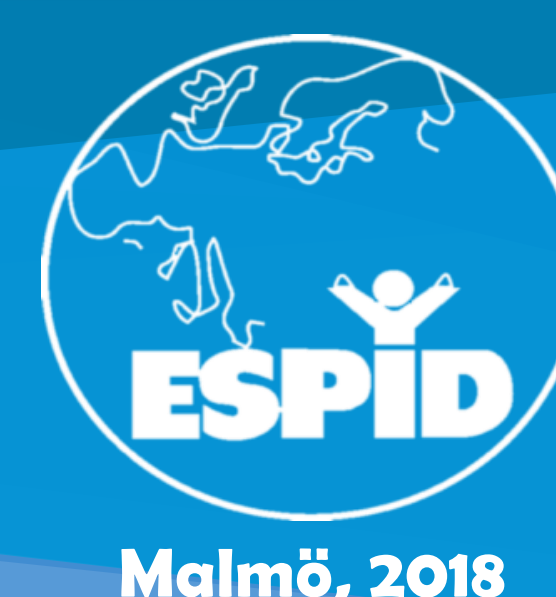

BACKGROUND - Yellow fever (YF) is an acute febrile disease, endemic in many regions of Brazil. Since 2005, there has been an expansion of the affected areas, with increasing number of cases despite yellow fever vaccination being offered as part of routine immunization in most states. In 2017/2018 a rapid increase in the number of confirmed human cases was observed in the states of Rio de Janeiro, São Paulo, and Minas Gerais. Since September 2017 the State of São Paulo has intensified mass vaccination campaign - as part of the world's largest vaccination campaign, to date, using fractional doses of YFV. The strategy aimed to create a blocking immunization ring, anticipating the eventual expansion of virus transmission, and assumed that there was no evidence of yellow fever transmission by the urban vector

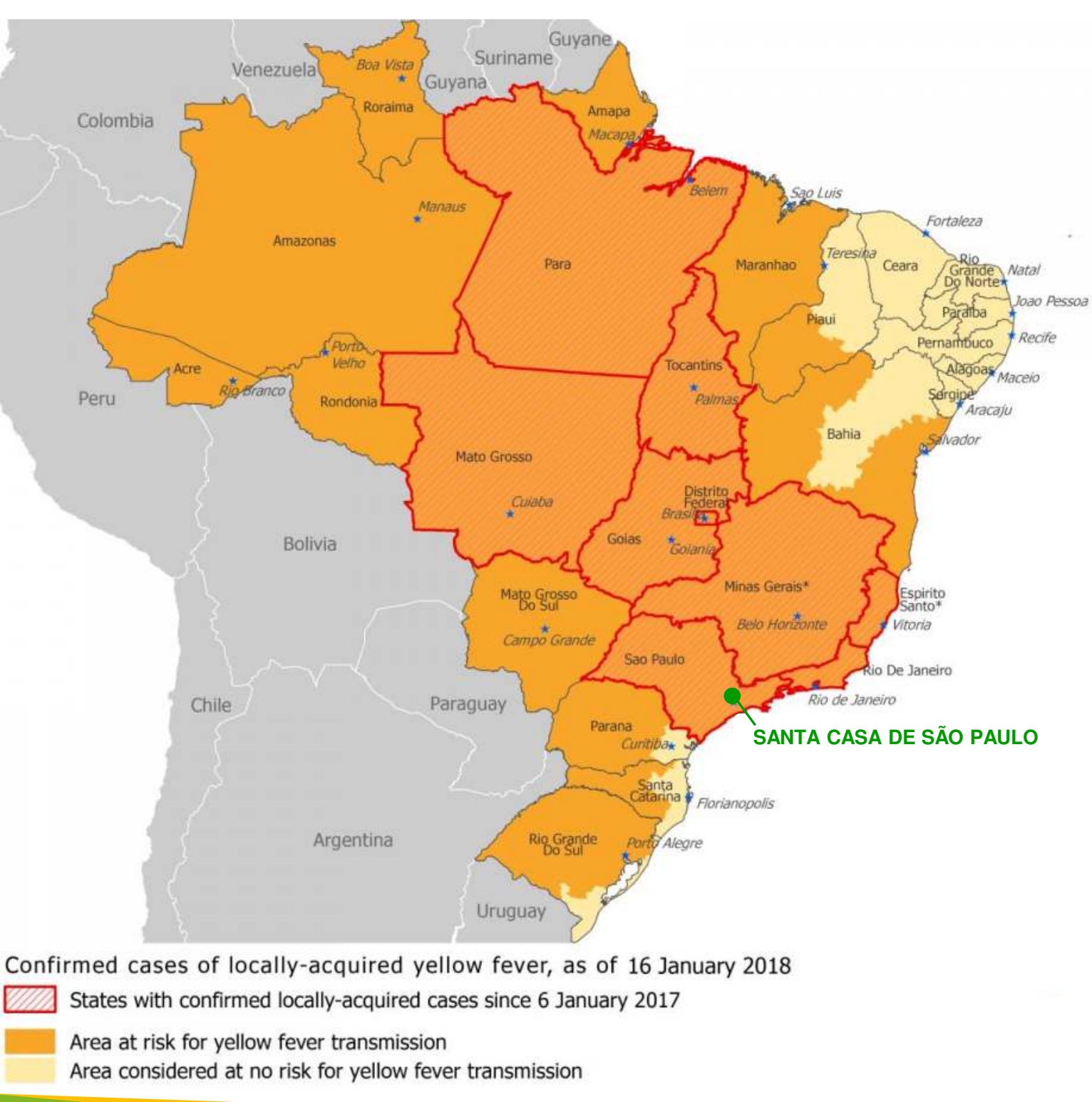

The main strategy for preventing the disease is through vaccination with a live attenuated virus vaccine (YFV). Despite generally safe for most target population, adverse events (AE) are more frequent following the first dosis. YF vaccineassociated neurologic disease (YEL-AND) comprise a rare but potentially severe spectrum of adverse events, presenting as acute meningoencephalitis, Guillain-Barré syndrome (GBS), and acute disseminated encephalomyelitis (ADEM). In Brazil, from 2007 to 2012, the overall rate of adverse neurological events after primary yellow fever vaccination (sub-strain 17DD) was 2 per 1,000,000 doses administered. The diagnostic criteria for YEL-AND is the presence of specific YF-IgM antibodies in the CSF, which is more sensible than RT-PCR.

CASE PRESENTATION SUMMARY - In 2017, 56 cases YEL-AND were reported in the state of São Paulo. We present two cases of meningoencephalitis after first dose of YFV (Table 1). Both patients presented specific YF-IgM antibodies in CSF. Clinical outcomes were favorable, without sequelae. Patients remain completely asymptomatic after 1 month of clinica follow-up.

Patient 1: a healthy 4 year old boy presented with headache, fever, dysarthria, decrease in muscular strength in the right upper limb and ataxic gait 25 days after having received his first YFV. Fever persisted for 5 days and he was hospitalized for 10 days.

Patient 2: a healthy 3 year old girl presented with headache, fever, irritability and partial seizures after 20 days after having received her first YFV. Patient was hospitalized for 10 days and required ICU care and ventilatory support. Total fever duration was 14 days.

\begin{tabular}{|c|c|c|}
\hline Age & PATIENT 1 & PATIENT 2 \\
\hline Gender & 4 years & 3 years \\
\hline Simptoms & Male & Female \\
\hline Time of symptoms onset after YFV & $\begin{array}{c}\text { Muscle strength decrease in the right } \\
\text { upper limb and ataxic gait }\end{array}$ & $\begin{array}{c}\text { Headache, fever, irritability and partial } \\
\text { seizures }\end{array}$ \\
\hline Fever & 25 days & 20 days \\
\hline Duration of hospitalization & 5 days & 14 days \\
\hline Specific YF-lgM antibodies in CSF & 10 days & 15 days \\
\hline
\end{tabular}

LEARNING POINT/ DISCUSSION - YFV adverse events are mostly mild, but severe episodes are expected at known rates. However, an important increase in the number of vaccine doses during emergency mass vaccination is expected to be followed by serious adverse events occurring at higher frequency (although retaining the aforementioned YEL-AND rate) than observed in routine immunization activities. This situation usually results from improved surveillance of $A E$ during campaigns, with higher sensitivity (and possibly lower specificity) for the identification and notification of such events. 